\title{
Microstructural Characterization of Manganese Oxides Supercapacitors based on Liquid-phase Exfoliated for Energy Storage Applications
}

\author{
Henrik Pettersson $^{1}$, Megan Canavan ${ }^{1}$, João Coelho ${ }^{2}$, Timothy Pennycook ${ }^{3}$ and Valeria Nicolosi ${ }^{1,2}$ \\ 1. School of Physics and CRANN, Trinity College Dublin, Dublin, Ireland. \\ 2. School of Chemistry and CRANN, Trinity College Dublin, Dublin, Ireland \\ 3. SuperSTEM, STFC Daresbury Laboratories, Warrington, United Kingdom.
}

Energy storage systems are becoming hugely important in today's world. More than ever, are we investing our time and resources into renewable energy production, such as solar, wind, hydra or even electric/hybrid electric vehicles with low $\mathrm{CO}_{2}$ emissions. Supercapacitors are currently bridging the gap between batteries and conventional capacitors, which is illustrated in figure 1. They have a higher power density than batteries and a higher energy density than electrolytic capacitors. Due to the large amount of surface area $\left(500-2000 \mathrm{~m}^{2} / \mathrm{g}\right)$ they can theoretically store up to tens to hundreds of farads per gram, which is much greater than a conventional capacitor. This is due to the charge being stored in the micropores at the electrode/electrolyte interface. However, they have lower energy densities, which mean they have very fast discharge time. [1-2]. Up until quite recently there has been only minor improvements in the development of supercaps and it is only since the discovery of new electrode materials and enhanced understanding of ion behaviour in small pores that it has improved so greatly and become such a topic of interest in recent years. This is due to the discovery of a whole new zoo of nanomaterials waiting to be explored.

The key to reaching high capacitance in supercaps is to have electrode nanomaterials possessing high surface area, high conductivity, porosity and chemically resistant to large voltage windows. This is why 2D layered materials have become such an attractive possibility for supercapacitors applications. They are merely a few nanometres in thickness and several microns wide; they have unique electrical properties and high surface area. At the moment the most well-known, talked about layered material is graphene.However there are many other layered materials which are being explored and are extremely important in the world of energy storage. These include transition metal dichalcogenides (TMDs), transition metal oxides (TMOs).

Manganese oxides belong to the Transition metal oxides family of compounds, which are generally considered the best candidates as electrode material for redox supercapacitors, possessing a wide range of oxidation states available for redox charge transfer.[3] $\mathrm{MnO}_{2}$ in particular is cheap and environmentally friendly, can also be used in neutral aqueous electrolytes and is a very accredited material used in bulk form in supercapacitors. Exfoliated nanosheets of $\mathrm{MnO} 2$ are here explored for the first time for the production of ultra-thin, flexible supercapacitors devices.

Nanoflakes are here produced by liquid-phase exfoliation of $\mathrm{MnO}_{2}$ powders in organic solvents such as Benzyl Alcohol.[4]

In this work transmission electron microscopy (TEM) was been used to determine the microstructure of the electrode material of the supercauacitors. The instruments used were FEI Titan 80-300 TEM and Nion Ultrastem ${ }^{\mathrm{TM}} 100$ Scanning TEM, both instruments equipped with EDX and EELS. 
The flakes obtained after exfoliation were small and possessing a lateral sizes of $5-20 \mathrm{~nm}$ approximately. The exfoliation yield was however satisfactory. Further investigation reviled that there were different manganese oxide stoichiometries present in the as-purchased raw powder, however liquid-phase exfoliation could ultimately be used to separate these phases.

References:

[1] U. C. Davis and A. Burke, Journal of Power Sources 91 (2000) 37-50,

[2] P. Simon and Y. Gogotsi, Nature Materials 7 (2008) 845-854, .

[3] X. Zhao et al, Nanoscale 3 (2011) 839-855

[4] J. N. Coleman et al., Science 331 (2011) 568-571

[7] A. Kuperman, and I. Aharon, Renewable \& Sustainable Energy Reviews 15 (2011) 981-992.

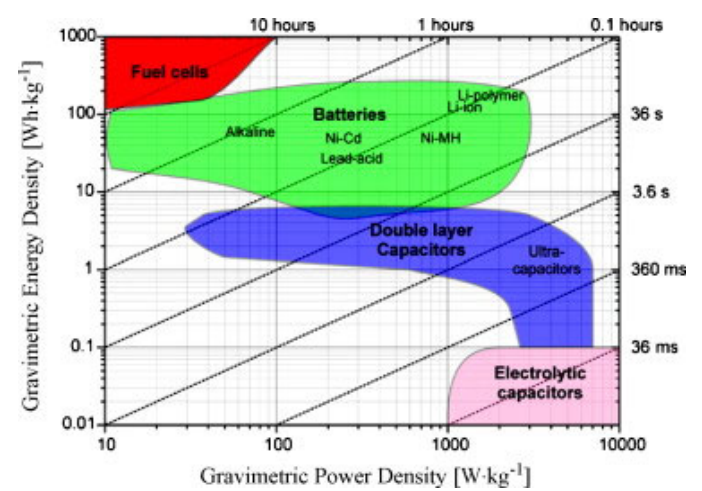

Figure 1. - Ragone plot showing specific energy density versus power density for various electrochemical energy storage devices [7]

a)

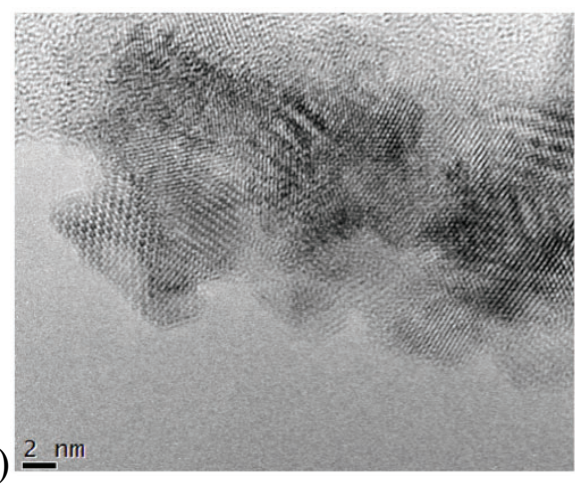

b)

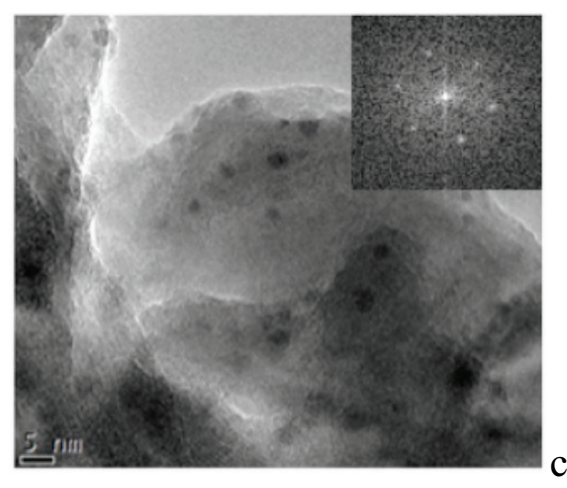

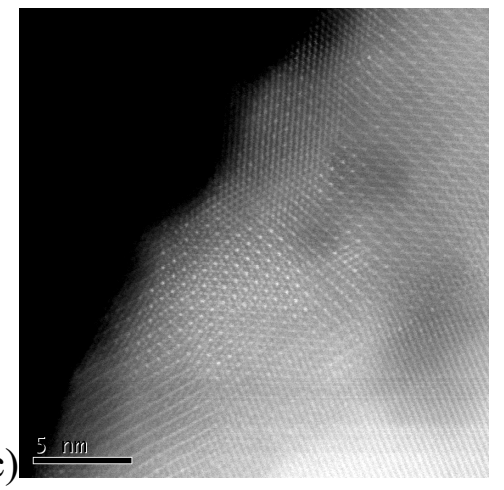

Figure 2. TEM images of $\mathrm{MnO}_{2}$ flakes obtained using Benzyl Alcohol under optimum sonication conditions, with initial concentration of a) $10 \mathrm{mg} / \mathrm{ml}$ and b) $2 \mathrm{mg} / \mathrm{ml} \mathrm{c)} \mathrm{STEM} \mathrm{image} \mathrm{of} \mathrm{MnO}_{2}$ where different orientations can be seen. 